\title{
GeSnOI mid-infrared laser technology
}

\author{
Binbin Wang ${ }^{1}$, Emilie Sakat ${ }^{1}$ Etienne Herth ${ }^{1}$, Maksym Gromovyi ${ }^{1}$, Andjelika Bjelajac ${ }^{1}$, Julien Chaste ${ }^{1}$, Gilles Patriarche , \\ Philippe Boucaud ${ }^{2}$, Frédéric Boeuf ${ }^{3}$, Nicolas Pauc ${ }^{4}$, Vincent Calvo ${ }^{4}$, Jérémie Chrétien ${ }^{4}$, Marvin Frauenrath ${ }^{5}$, \\ Alexei Chelnokov ${ }^{5}$, Vincent Reboud ${ }^{5}$, Jean-Michel Hartmann ${ }^{5}$ and Moustafa El Kurdi@ ${ }^{1 凶}$
}

\begin{abstract}
GeSn alloys are promising materials for CMOS-compatible mid-infrared lasers manufacturing. Indeed, Sn alloying and tensile strain can transform them into direct bandgap semiconductors. This growing laser technology however suffers from a number of limitations, such as poor optical confinement, lack of strain, thermal, and defects management, all of which are poorly discussed in the literature. Herein, a specific GeSn-on-insulator (GeSnOl) stack using stressor layers as dielectric optical claddings is demonstrated to be suitable for a monolithically integration of planar Group-IV semiconductor lasers on a versatile photonic platform for the near- and mid-infrared spectral range. Microdisk-shape resonators on mesa structures were fabricated from $\mathrm{GeSnOl}$, after bonding a $\mathrm{Ge}_{0.9} \mathrm{Sn}_{0.1}$ alloy layer grown on a $\mathrm{Ge}$ strain-relaxed-buffer, itself on a Si(001) substrate. The GeSnOI microdisk mesas exhibited significantly improved optical gain as compared to that of conventional suspended microdisk resonators formed from the as-grown layer. We further show enhanced vertical out-coupling of the disk whispering gallery mode in-plane radiation, with up to $30 \%$ vertical out-coupling efficiency. As a result, the GeSnOl approach can be a valuable asset in the development of silicon-based mid-infrared photonics that combine integrated sources in a photonic platform with complex lightwave engineering.
\end{abstract}

\section{Introduction}

Low-cost and CMOS-compatible Si-based photonic technologies, like Silicon-On-Insulator (SOI), has enabled significant advances for on-chip optical processing in the near-infrared (IR) wavelength range, especially for highspeed detection and modulation of optical signals ${ }^{1}$. One of its major drawbacks, though, is the lack of monolithically integrated group-IV lasers. Indeed, group-IV alloys are indirect bandgap semiconductors. To compensate for the lack of such laser sources, strong efforts were devoted these past few years to the integration of III-V compounds with high lasing performances to boost silicon photonic technologies ${ }^{2}$. It was particularly true for telecom applications in the near-infrared wavelength range. So far, III-V lasers are the most standard and reliable light

\footnotetext{
Correspondence: Moustafa El Kurdi (moustafa.el-kurdi@c2n.upsaclay.fr) 'Université Paris-Saclay, CNRS, C2N, 10 boulevard Thomas Gobert, 91120 Palaiseau, France

Université Côte d'Azur, CNRS, CRHEA, Rue Bernard Grégory, 06905 SophiaAntipolis, France

Full list of author information is available at the end of the article
}

sources on silicon despite their high manufacturing cost and their complex integration in $\mathrm{Si}$ CMOS-compatible manufacturing processes ${ }^{3,4}$. GeSn semiconductor alloys, with a direct bandgap for tin contents larger than 7\% (for strain-free materials) ${ }^{5}$ and which are compatible with large-scale and low-cost silicon processing and manufacturing tools, are promising for low-cost lasers ${ }^{6-8}$. In addition, GeSn alloys have a narrow bandgap as compared to $\mathrm{Ge}$ and are thus suitable to shift the photonic wavelength from near-IR to mid-IR where many application domains exist: biochemical detection, gas monitoring, and thermal imaging. Integrating $\mathrm{GeSn}$ on silicon opens up new application fields for Si photonics ${ }^{9,10}$.

In practice, the epitaxial growth of GeSn on silicon is quite challenging. The very low thermal equilibrium solubility of $\mathrm{Sn}$ in $\mathrm{Ge}$, of only $1 \%$, requires the development of metastable growth methods to increase the Sn content above $7 \%$. Moreover, the very high lattice mismatch between GeSn and silicon makes their growth tricky. The use of Ge strain-relaxed buffer (Ge SRB) on silicon is the only approach used so far yielding high

\section{(c) The Author(s) 2021}

(c) Open Access This article is licensed under a Creative Commons Attribution 4.0 International License, which permits use, sharing, adaptation, distribution and reproduction in any medium or format, as long as you give appropriate credit to the original author(s) and the source, provide a link to the Creative Commons license, and indicate if changes were made. The images or other third party material in this article are included in the article's Creative Commons license, unless indicated otherwise in a credit line to the material. If material is not included in the article's Creative Commons license and your intended use is not permitted by statutory regulation or exceeds the permitted use, you will need to obtain permission directly from the copyright holder. To view a copy of this license, visit http://creativecommons.org/licenses/by/4.0/. 
enough material quality for lasing. However, it still faces major issues ${ }^{11-13}$. For example, the active GeSn layers are usually grown beyond their critical thickness for plastic relaxation of the compressive strain at the $\mathrm{GeSn} / \mathrm{Ge}$ interface $^{14}$. Compressive strains are unfavorable since they worsen the optical gain properties of GeSn alloys, and, specifically, their sustainability with increasing temperature. Indeed, they reduce the energy barrier between the direct $E_{\Gamma}$ and indirect $E_{\mathrm{L}}$ conduction band valleys (the so-called material directness parameter) ${ }^{15}$. Compressive strain in pseudomorphically grown GeSn alloys can turn, even for high $\mathrm{Sn}$ contents, the bandgap from direct to indirect, preventing gain and lasing ${ }^{16}$. Moreover, the relaxation of this compressive strain during the growth of really thick layers results in the formation of a dense array of misfit dislocations network close to the interface, at the bottom of the optically active region ${ }^{5,8}$. Thus, many strategies have been employed these past few years to address this compressive strain issue while attempting to reach the highest material quality possible: growth of thick GeSn layers with gradually increasing tin content in order to limit the propagation of misfit defects into the bulk of the GeSn layer, use of SiGeSn barriers to confine the carriers away from the interfacial defects or, even, multi-quantum wells ${ }^{11,17-21}$. All of those methods are based on in situ strain and defects management and have thus limited flexibility due to metastable growth mechanism constraints.

In this framework, the active injection of tensile strain into GeSn, via external stressor layers, is theoretically and experimentally known to offer further degrees of freedom to tune the strain and consequently the electronic band structure $^{22}$. It also opens up new possibilities of enhancing gain properties and tuning the laser wavelength ${ }^{23,24}$. Mainstream technological approaches for strain management call upon external stressor layers such as $\mathrm{SiN}^{25-27}$ or mechanical pulling of microbridges by external pads ${ }^{28-30}$. They were mainly used for pure germanium, up to now. In both cases, layers should be suspended in order to increase the tensile stress injection and reach high enough optical confinement. Indeed, blanket GeSn on Ge SRB structures suffers from a low optical index contrast between GeSn and $\mathrm{Ge}$, resulting in low optical confinement factors in the active region $^{31}$. The active layer thickness has then to be increased in order to reach values in the range of the operating wavelength, e.g. $1 \mu^{6}$, making external tensile strain injection more challenging. Suspended microdisk (MD) cavities or microbridges were thus the main structures evaluated up to now for such strained laser devices $^{22,32}$.

In this work, we show that GeSnOI stacks obtained through the bonding of GeSn active layers tackle all the above-mentioned issues: lattice mismatch interface defects management, compressive/tensile strain management, and optical confinement. This is demonstrated through a systematic comparison of two structures with equivalent optical confinements: a suspended MD cavity fabricated from a GeSn layer on a Ge SRB, as in the existing literature, and another one, fabricated using the GeSnOI approach, with the use of specific SiN stressor films as insulating layers and with a simple disk-shape mesa as the cavity. The SiN layers used for tensile strain engineering yielded high index contrast with GeSn, which was required for optical confinement. After the bonding of the GeSn layer, the dense array of misfit dislocations close to the $\mathrm{GeSn} / \mathrm{Ge}$ interface was removed thanks to a simple etching step, resulting in a better active layer quality that should result in an improved carrier injection efficiency. Another advantage offered by such bonded structures was the possibility of having the GeSn layer standing on an aluminum layer, resulting in a better heat dissipation ${ }^{27,33}$. Moreover, such disk cavities being bonded to the substrate without any under-etching, it was also possible to downscale their diameters to $3 \mu \mathrm{m}$, which was not feasible with as-grown GeSn-MD (lowest achievable diameters of $5 \mu \mathrm{m}$ ) for mechanical and thermal robustness reasons (the $\mathrm{Ge}$ pedestals cannot be too thin). Last but not least, the MDshape mesa cavity obtained with this new approach also offered the possibility of managing the whispering gallery modes (WGM) in-plane radiation that made the collection of the laser light tricky. A specific design with circular diffraction grating around the MD was proposed to redirect the emission pattern vertically without changing the MD design or the lasing characteristics.

\section{Results}

We start with a presentation of the two kinds of structures. Both were based on a 500-nm-thick GeSn layer with $10.5 \%$ of $S n$ grown on a $2.5-\mu$ m-thick Ge SRB, itself on a $200 \mathrm{~mm} \mathrm{Si} \mathrm{(001)} \mathrm{wafer} \mathrm{(Fig.} \mathrm{1a).} \mathrm{The} \mathrm{growth} \mathrm{was}$ performed in an industrial reduced-pressure chemical vapor deposition (RP-CVD). The as-grown GeSn layer had a residual compressive strain of $-0.5 \%$ as estimated from X-ray diffraction reciprocal space mapping ${ }^{5}$. For such GeSn layer on Ge SRB, the critical thickness is around $100 \mathrm{~nm}$. A dense array of misfit dislocations loops occurs up to a depth of typically $100 \mathrm{~nm}$ from the GeSn/ Ge interface, can clearly be seen by cross-sectional transmission electron microscopy (X-TEM) (Fig. 1b), a zoomed view is provided in the SI (Fig. S1).

The optical index contrast is low in such a GeSn on the Ge SRB stack, resulting in a poor mode overlap with the active GeSn layer (Fig. 1a). The transverse electric (TE)polarized propagating wave indeed has an overlap of only $37 \%$ with the GeSn active layer and its electrical field maximum is close indeed to the dislocated interfacial region. Similarly, a blanket GeSn on Ge stack provides a very weak transverse magnetic (TM)-polarized light 


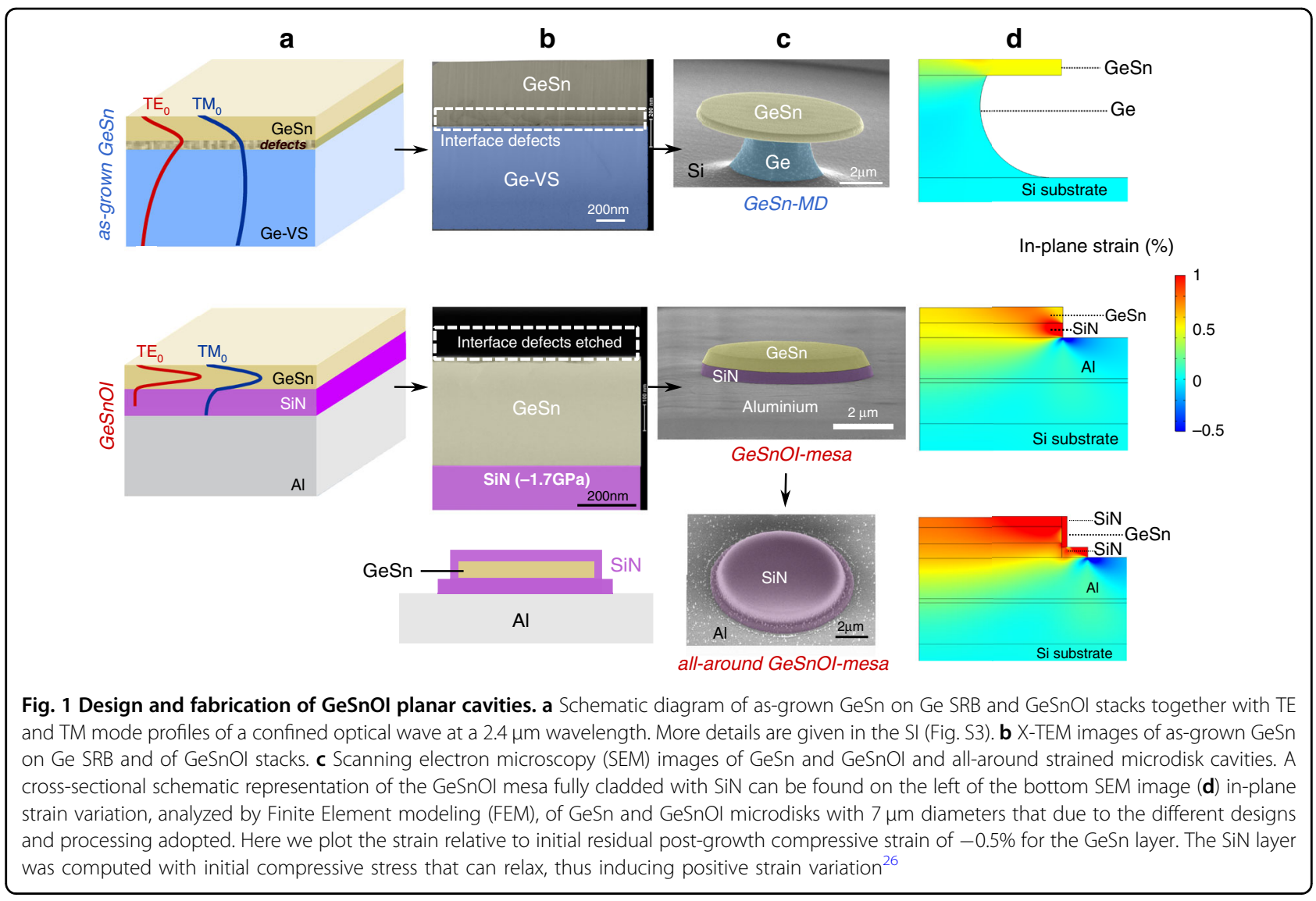

confinement, with an overlap of only $11 \%$ with the GeSn active layer. To circumvent such bad optical confinements, an isotropic under-etching of the Ge SRB is generally performed in order to obtain suspended GeSn-MD laser devices (Fig. 1c). In this configuration, the WGM situated at the edges of the disk experiment a GeSn/air optical index contrast which yields optical confinement factors of $95 \%$ and $78 \%$ for TE and TM, respectively.

For the MD-shape mesa laser cavity, the approach was different: a piece of the very same GeSn on Ge SRB was bonded on a host Si substrate coated with aluminum and SiN layers to obtain the GeSnOI structure shown in Fig. $1 \mathrm{~b}, \mathrm{c}$ (process detailed in the section A of the SI). The aluminum layer acted as a heat sink while the SiN layer acted as a stressor and provided optical confinement. After bonding, the Si substrate used to grow the Ge SRB was removed by selective etching. The Ge SRB and defects at the $\mathrm{GeSn} / \mathrm{Ge}$ interface were then removed by another etching step. The final GeSn layer was then thinned from the as-grown $500 \mathrm{~nm}$ down to $400 \mathrm{~nm}$, to get rid of the defective interfacial layer, which was then on top, and benefit from improved crystalline quality. The GeSn/SiN index contrast was high enough to provide excellent optical confinement for TE and TM-polarized modes: optical confinement factors were $93 \%$ for TE and $77 \%$ for
TM, e.g., values very close to that in GeSn MDs (Fig. 1c). This enabled us to suppress the impact of confinement on the optical gain when comparing the lasing performances of conventional $\mathrm{GeSn} / \mathrm{Ge} \mathrm{MDs}$ and MD-shape mesas based on bonded GeSnOI. Confining the modes in a few hundreds of nm-thick-GeSn layer was suitable for homogeneously distributed external tensile strain injection (Fig. 1d). The SiN insulator was initially compressively stressed, by typically $-1.7 \mathrm{GPa}$. The strain was partly released and transferred to the GeSn layer after patterning the GeSnOI layers into MD-shape mesas, as shown by the FEM analysis. The GeSn layer had an initial compressive strain of $-0.5 \%$ which vanished after the bottom SiN layer had partially released its built-in stress. This led to a very moderately tensile-strained structure (Fig. 1d). To further increase the tensile strain, an additional top SiN stressor layer can be deposited, resulting in an all-around structure. The tensile strain is then homogeneously distributed in the GeSn disk, as shown in Fig. 1d. An SiN stressor layer could also be deposited on top of the GeSn-on-Ge SRB MD, as proposed recently ${ }^{24,34}$. The distributed tensile strain would be very inhomogeneous, then, which would not be favorable for lasing, as discussed in the SI (section F). Raman analysis of the strain (Fig. 2a) was performed using the strain and alloy potentials of 

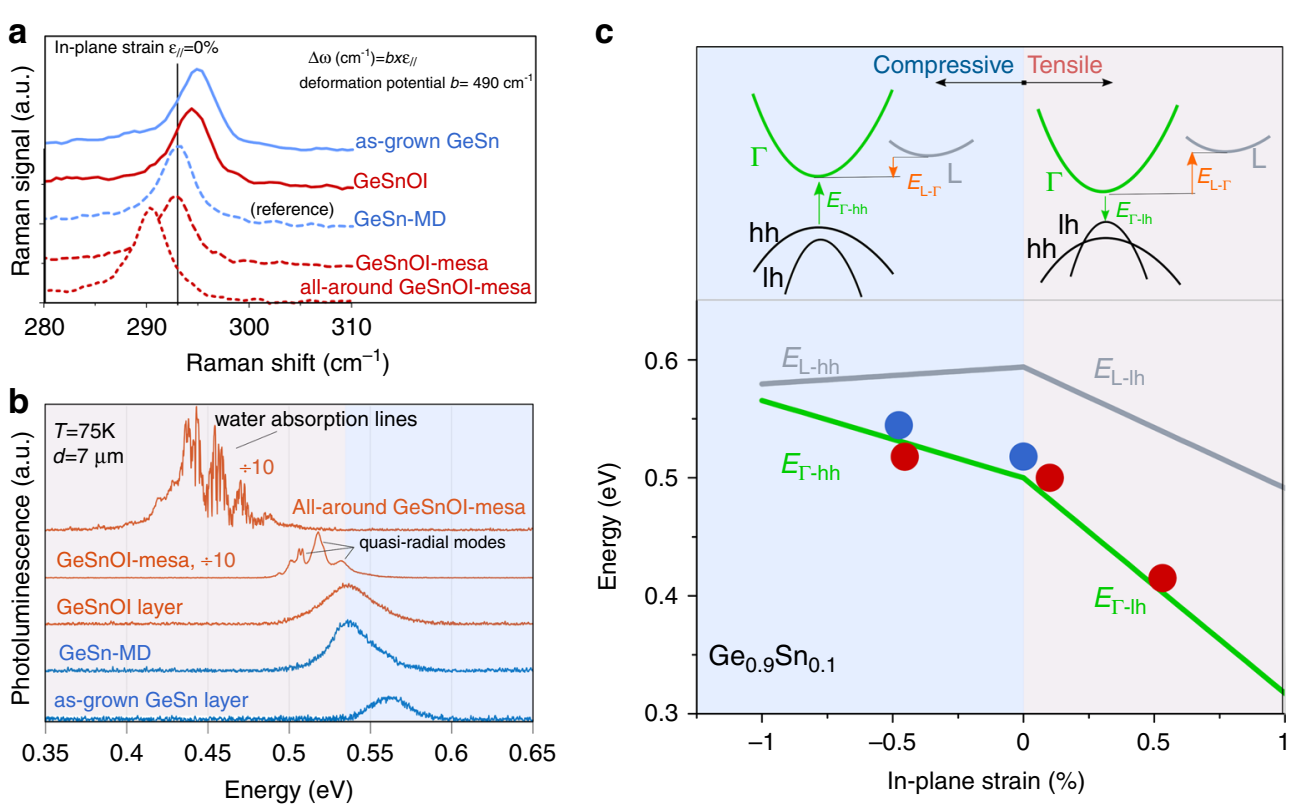

Fig. 2 Optical analysis of strain. a Raman spectra of LO-phonon vibrations in the blanket, as-grown GeSn 10.5\% on Ge SRB stacks, blanket GeSnOI stacks, GeSn-on-Ge SRB microdisks, GeSnOI mesas, and all-around GeSnOl mesas. The diameter of disks and mesa was $7 \mu$ m. b Photoluminescence spectra of GeSn layers as in (a) excited with cw optical pumping. c Direct and indirect bandgap energies of GeSn as a function of strain. Direct bandgap energies from PL measurements appear as blue circles for the as-grown GeSn 10.5\% on Ge SRB stack and the GeSn-on-Ge SRB microdisks. Red circles show the PL energies for the blanket GeSnOl stack, the GeSnOI mesa, and the all-around GeSnOl mesa (from left to right). Full lines are calculated direct bandgap (green) and indirect bandgap (gray) energies as functions of strain using model as described in ref. ${ }^{5}$. The top picture is a schematic scenario of band structure for compressive and tensile-strained GeSn

ref. ${ }^{5}$. To identify the influence of strain on the band structure, we have performed a continuous wave $(\mathrm{cw})$ photoluminescence (PL) analysis at $75 \mathrm{~K}$ under $1550 \mathrm{~nm}$ wavelength optical pumping. Different configurations, blanket GeSn on Ge SRB stacks, GeSn-on-Ge SRB MDs, blanket GeSnOI stacks, GeSnOI mesas and all-around GeSnOI mesas, were studied under the same experimental conditions, as shown in Fig. 2.

The suspended GeSn MDk has a PL emission redshifted by $25 \mathrm{meV}$ with respect to the PL emission from the blanket, unprocessed GeSn layer, which is due to compressive strain relaxation through the under-etching of the Ge SRB at the disk edges. The continuous wave PL spectrum of the GeSnOI mesa has red-shifted by $18 \mathrm{meV}$ only with respect to the PL spectrum of the strain-relaxed GeSn-MD (without SiN stressor). This indicates that the mesa is very weakly tensile-strained, as confirmed by Raman analysis which gives only around $0.1 \%$ of in-plane strain, in line with FEM analysis. On the other hand, the all-around GeSnOI disk-shape mesa has a $100 \mathrm{meV}$ redshifted emission compared to the GeSn-MD. An injected equivalent biaxial tensile strain of $0.55 \%$ was measured with Raman spectroscopy, once again in good agreement with FEM analysis. We have plotted in Fig. 2c the bandgap energies extracted from PL spectra as a function of the strain measured by Raman spectroscopy. Full curves are theoretical direct bandgap $\Gamma-\mathrm{hh}(\Gamma-\mathrm{lh})$ and indirect bandgap L-hh (L-lh) energies as functions of compressive (or tensile) $\operatorname{strain}^{5}$. A quite good agreement is obtained with experimental results (points in blue or red). The $\mathrm{SiN}$ stressor thus enabled us to modify the strain state of our GeSn $10.5 \%$ alloy, from a $-0.5 \%$ compressive state to a $0.55 \%$ tensile state, e.g., a shift by $\sim+1 \%$ of the strain. Band structure engineering was thus feasible without changing the Sn content.

To conclude this section, let us summarize the improvements provided by MD-shape mesas etched in bonded $\mathrm{GeSnOI}$ stacks as compared to suspended $\mathrm{GeSn}$ MDs. First, a GeSnOI disk-shape mesa has no interfacial defects anymore, while the suspended GeSn-MD still has defects at the pedestal's GeSn/Ge interface. Second, the bonded structure is not under-etched and incorporates an aluminum layer which acts as a heat sink, enabling a better thermal management. The optical confinement factor of optical modes with the active GeSn layer is as good in GeSnOI mesas than in under-etched structures suspended in the air. Finally, a full cladding of the GeSn mesa with $\mathrm{SiN}$ insulator layers yields high levels of homogeneously distributed tensile strain, something which is not really feasible with a suspended GeSn-MD. In the latter case, the only practical option is to add a stressor on the top of the suspended GeSn layer ${ }^{24,34}$. Such 

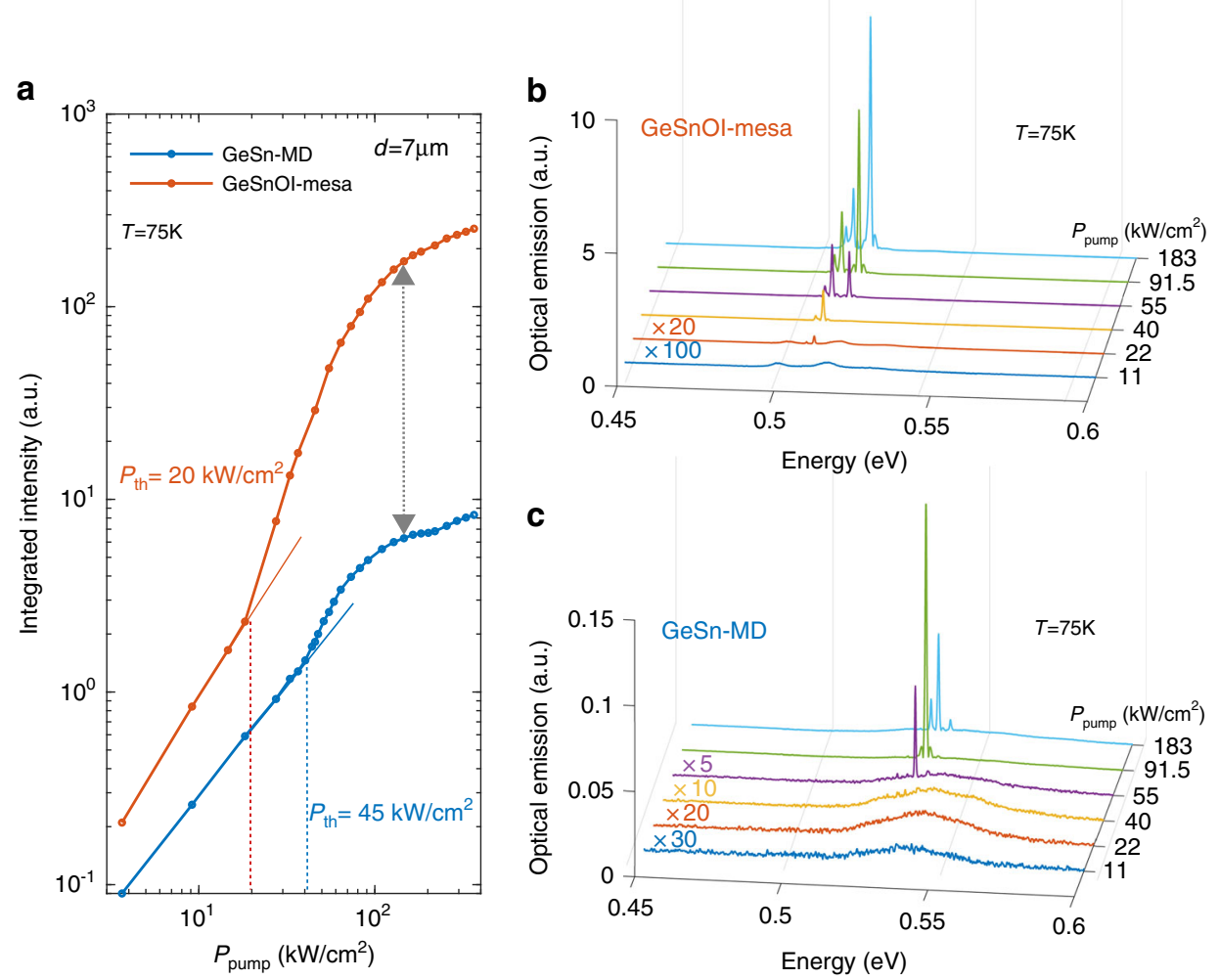

Fig. $3 \mathrm{GeSnOI}$ mesa versus as-grown GeSn microdisk laser. a Light-Light $(L-L)$ curves of $7 \mu$ m diameter GeSn microdisks and GeSnOl mesas with 360-nm-thick GeSn at $75 \mathrm{~K}$ under pulsed pumping (b) and $\mathbf{c}$ PL spectra at $75 \mathrm{~K}$ for various pulsed excitation powers for the GeSnOl mesa and the GeSn-MD, respectively. Excitation levels are given by the peak power densities

configuration results in an inhomogeneous tensile strain which is not convenient for lasing (see the SI section). Let us now show, in the next sections, how these improvements enabled us to enhance lasing performances.

Lasing performances were compared for the same $7 \mu \mathrm{m}$ diameter mesas and MDs. The GeSnOI mesa has an active layer thickness of $360 \mathrm{~nm}$, while the GeSn-MD active layer thickness is $500 \mathrm{~nm}$. The analysis of the MD emission was performed with a $1550 \mathrm{~nm}$ wavelength pulsed optical pumping with a pulse width of $3.5 \mathrm{~ns}$ and a $25 \mathrm{MHz}$ repetition rate. This illumination condition yielded high excitation densities while avoiding any significant thermal heating (SI section C). As shown in Fig. 3a-c, we observed clear lasing for mesas and MDs in the pulsed excitation regime. However, a two times lower threshold was obtained with the GeSnOI mesa $(20 \mathrm{~kW} /$ $\left.\mathrm{cm}^{2}\right)$, as compared to the GeSn-MD $\left(45 \mathrm{~kW} / \mathrm{cm}^{2}\right)$. In addition, the GeSnOI mesa had a peak intensity above lasing threshold which was 60 times higher than that of the GeSn-MD (Fig. 3b, c), while the spontaneous emission below threshold was typically four times more intense (Fig. 3a). We can note that the PL enhancement, below threshold with GeSnOI mesa due to the presence of the reflective $\mathrm{Al}$ layer with respect to the GeSn-MD, can be estimated to be 1.7, which is lower than the observed enhancement. Furthermore (i) the GeSnOI mesa absorbs less efficiently, by a factor of 1.7, the incident pump beam (as discussed below); (ii) the GeSnOI active layer is a factor of 1.4 thinner than the GeSn-MD and thus has less emitters in its volume. We can thus assume that the improved PL signal below the threshold, despite the given above consideration, goes to the sense that carrier losses from non-radiative recombination process are partly reduced with the GeSnOI mesa, which could also partly explain such gain dynamic and threshold improvements $^{35,36}$. In previous work, we already demonstrated that the power density threshold in suspended GeSn MDs was one order of magnitude lower after the partial removal of interfacial defects from the MD edge region during the Ge under-etching ${ }^{5}$. However, interface defects above the Ge pillars were still present. Here, defects in GeSnOI mesas were removed from the whole surface, most likely explaining why power density thresholds were further reduced. The tensile strain in the GeSnOI mesa was small (only around $0.1 \%$ ), with a limited impact on band structure and gain. We can note that the presence of the reflective $\mathrm{Al}$ layer, in the case of GeSnOI stack, may induce an enhancement of the pump beam absorption by 
reflection effect from the bottom $\mathrm{Al}$ layer. However depending on the SiN layer thickness, one may have constructive or destructive interference between the incident and the reflected pump beam, inside the active $\mathrm{GeSn}$ region. In our specific case, the GeSnOI stack dimensions were not favorable, and the GeSn active layer is positioned at a node of the electrical field distribution along the $\mathrm{GeSn} / \mathrm{SiN} / \mathrm{Al}$ stacking. We estimated that in this configuration, the absorbed power of incident pump is significantly larger here for the GeSn-MD case, by typically a factor 1.7. The absorption coefficient is estimated to be $30 \%$ for the GeSn-MD while the GeSnOI mesa have an absorption coefficient of $18 \%$. We can thus assume that the improved lasing thresholds were not induced by enhanced pump power absorption in the GeSnOI mesa. However further improvement of the laser characteristics could be reached thanks to appropriate design of the GeSnOI stack, for example, by using an SiN layer thickness of $600 \mathrm{~nm}$, instead of $400 \mathrm{~nm}$ as in the present case. With such SiN layer thickness the GeSn active layer would thus be positioned favorably at the antinode of the electrical field distribution of the pump such that one could reach a strongly improved efficiency of the pump absorption, typically by a factor of 4.5 . In this case the absorption coefficient of the pump could reach $80 \%$. It is additionally important to note here that the quality $(Q)$ factor of the GeSnOI mesa is two times lower than that of the GeSn-MD, which should have increased the GeSnOI mesa's lasing threshold. It is however lower than that of the GeSn-MD, illustrating the importance of defects removal to improve the gain. One can also observe in Fig. $2 \mathrm{~b}$ that the GeSnOI mesa features multimodal lasing, which is the signature of spatial and spectral broadening of the gain, whereas the GeSn-MD has mostly a monomode lasing signature.

This can be explained by the presence of the Ge pedestal in the GeSn-MD. Indeed, the edges of the pedestal are $1.5-2 \mu \mathrm{m}$ away from the MD periphery, resulting in optical losses for higher-order radial modes, i.e., those which have a higher overlap of the optical field with the pedestal. The $Q$-factor of these modes decreases and they are not able to lase. On the contrary, the same diameter GeSnOI mesa has the whole surface isolated from the substrate by the bottom SiN layer. Therefore, the higherorder radial modes do not incur any pedestal losses. This is also confirmed by the PL spectra of the GeSnOI mesa below threshold, with the presence of high-order radial mode resonances (denoted as quasi-radial modes in Fig. $2 b)$, which were not observed for the GeSn-MD. The multimodal features thus could also be explained by a higher spatial gain broadening with the GeSnOI mesa.

The GeSnOI mesa gain also persists at higher temperature. Lasing spectra above threshold (Fig. 4a) along with $\mathrm{L}-\mathrm{L}$ curves and lasing thresholds (Fig. 4b, c) at different temperatures clearly show the improvement when switching from $7 \mu \mathrm{m}$ diameter GeSn MDs to GeSnOI mesas, with maximum lasing temperatures of 80 and $135 \mathrm{~K}$, respectively. One can estimate, from threshold dependence with temperature of Fig. 4c, a $T_{0}$ value of $40 \mathrm{~K}$. This value can be associated with activation of nonradiative recombination process, and intervalley scattering that weaken the optical gain when increasing the temperature. The slope efficiency is as well better above threshold for GeSnOI mesas and this feature is attributed to higher quantum efficiency associated with better carrier distribution in the gain region.

Then, different diameter GeSn MDs and GeSnOI mesas were systematically studied to compare their lasing threshold and their maximum lasing temperature. Thresholds at $75 \mathrm{~K}$ are provided in Fig. $4 \mathrm{~d}$ and maximum lasing temperatures in Fig. 4e, both as functions of the MDs diameter. We reproducibly obtained lower thresholds and higher maximum lasing temperature for GeSnOI mesas than for GeSn MDs.

A heat dissipation study was conducted for suspended GeSn MDs (see SI section C). It showed that thermal cooling strongly depended on the MD geometry. We did not observe lasing for MD diameters smaller than $6 \mu \mathrm{m}$ with therefore very narrow Ge pedestals. Since the under-etching was the same for all diameters, MDs with the largest diameters had larger pedestals and evacuated heat more effectively. For GeSn-MD diameters between 7 and $9 \mu \mathrm{m}$, the temperature increase upon optical injection should be around $15 \mathrm{~K}$ for a $200 \mathrm{~kW} / \mathrm{cm}^{2}$ excitation density in a pulsed regime, as used in the current experiments. Yet, MDs were not able to reach lasing at temperatures higher than $85 \mathrm{~K}$. This maximum lasing temperature is similar to that in a previous work where MDs were fabricated from the very same GeSn $10.5 \%$ layer with equivalent disk diameters but smaller undercuts $(1.5 \mu \mathrm{m}$ instead of $2 \mu \mathrm{m}$ here). Such reduced lasing temperatures are likely due to limited heat dissipation and gain decrease as the temperature increases.

In GeSnOI mesas, the maximum lasing temperature is $140 \mathrm{~K}$, i.e., $55 \mathrm{~K}$ higher than in suspended GeSn MDs. Such an improvement is attributed to a higher crystalline quality, i.e., a lower number of non-radiative recombination channels making the temperature dependence of gain more robust, and to the increased heat dissipation. With such mesa structures, disk diameters can be shrunk down to $3 \mu \mathrm{m}$ without degrading thermal dissipation upon optical pumping. A reduced size seems to help heat dissipation. Indeed, as seen in Fig. 4e, the lowest diameter mesa has the highest lasing temperatures. Such $3 \mu \mathrm{m}$ diameter MD mesa also features the smallest lasing thresholds, only $12 \mathrm{~kW} / \mathrm{cm}^{2}$ at $75 \mathrm{~K}$ and $40 \mathrm{~kW} / \mathrm{cm}^{2}$ at $140 \mathrm{~K}$ (Fig. 5a, b). 


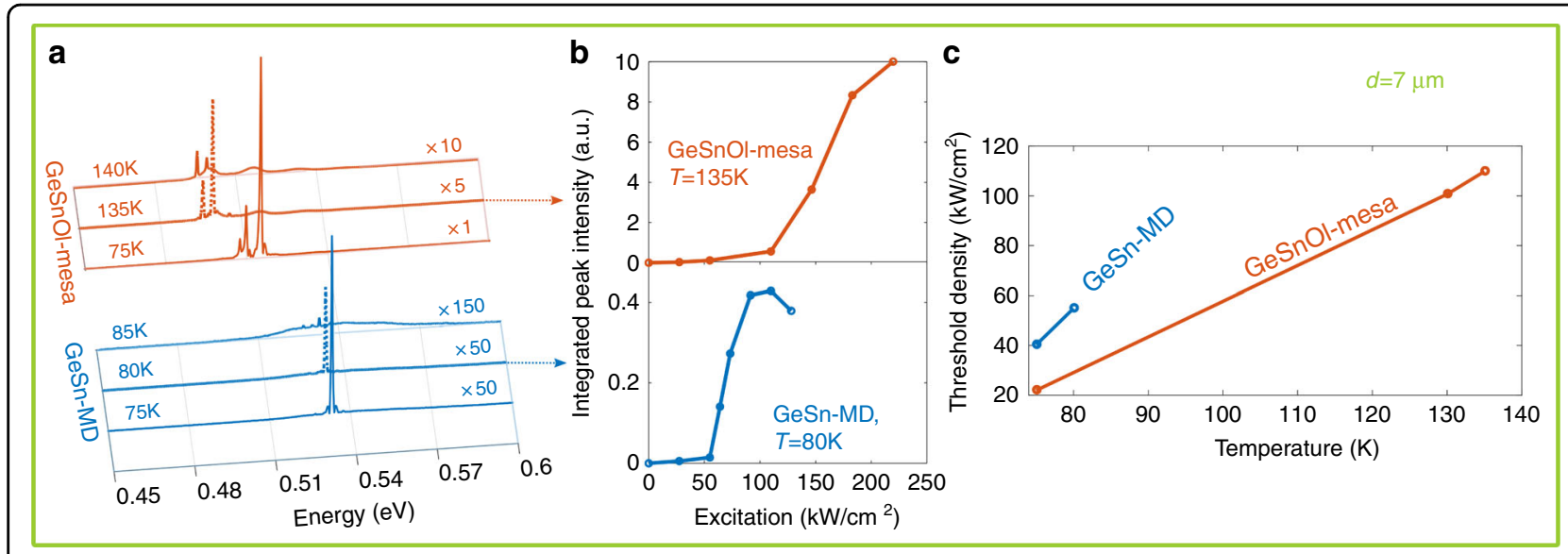

d

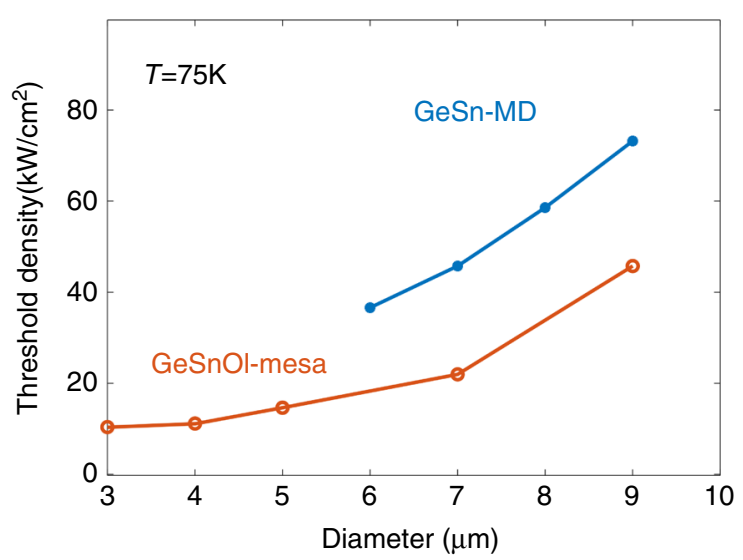

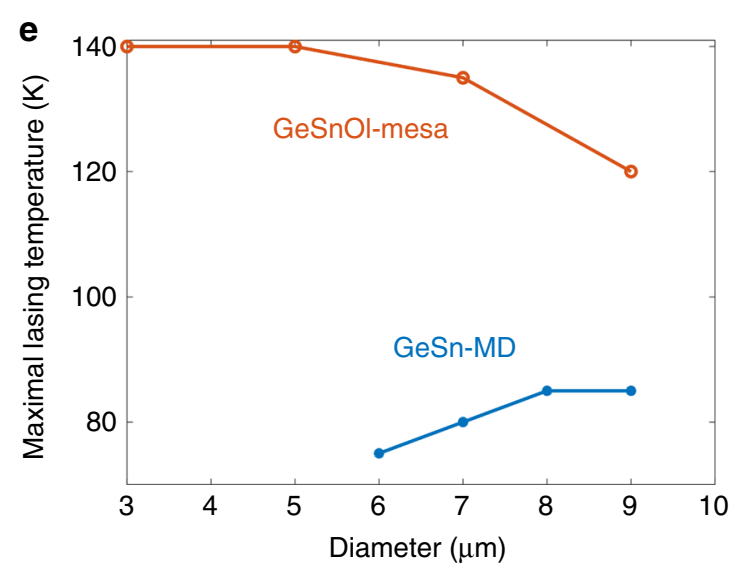

Fig. 4 Improved lasing performances with GeSnOl. a Pulsed PL spectra of $7 \mu$ m diameter GeSn microdisks and GeSnOl mesas with 360-nm-thick GeSn above the threshold at $75 \mathrm{~K}$ (solid curves), at maximum lasing temperatures (dotted curves) and at temperatures $5 \mathrm{~K}$ higher (dashed curves). The laser intensities in GeSn MDs and GeSnOI mesas are the highest for 91.5 and $183 \mathrm{~kW} / \mathrm{cm}^{2}$ excitation power densities, respectively. $\mathbf{b} \mathrm{L}-\mathrm{L}$ curves for GeSn MDs and GeSnOI mesas at their maximum lasing temperature, 80 and $135 \mathrm{~K}$, respectively. c Temperature dependence of threshold densities for $7 \mu \mathrm{m}$ diameter GeSn MDs and GeSnOl mesas. d Power density thresholds at $75 \mathrm{~K}$ for different diameter GeSn-MD and GeSnOl mesa lasers. e Highest lasing temperatures for different diameter GeSn MDs and GeSnOl mesas

Lasing thresholds decrease with the mesa or MD diameters. This was expected as smaller volume cavities have larger spontaneous coupling factors with the optical modes $^{36,37}$. Here, the coupling factor $\beta$ was found to be $10^{-3}$ from a fit of $\mathrm{L}-\mathrm{L}$ curve of the lasing mode using laser rate equations (Fig. 5b). The $3 \mu \mathrm{m}$ smallest diameter mesa indeed features single-mode lasing at $0.525 \mathrm{eV}$ at $75 \mathrm{~K}$, which is attributed to a $\mathrm{TE}_{10,1}$ WGM resonance. The sharp linewidth decrease at threshold for this mode allows for an estimate of an equivalent $Q$-factor value at transparency of typically 1000 . A linewidth increase is observed above threshold due to thermal effects. This $Q$-factor value at transparency has to be compared with modeled values of $Q$-factor around 10,000-13,000 (see Fig. 6). Note that the measurements are performed in pulsed excitation and the linewidth might vary over the pulse duration, thus inducing larger experimental linewidth than modeled ones in the framework of steady-state regime. The differences may also stem from fabrication imperfections such as surface roughness of the GeSnOI layer after bonding (Fig. S1) as well as the sidewall roughness of the mesas. The modeled free spectral range is equal to $35 \mathrm{meV}$ for the fundamental radial mode $n=1$ in such small diameter mesas, which is of the same order of the spontaneous emission broadening. It is thus expected that such a small cavity has a reduced number of modes that overlap with gain, resulting in single-mode lasing as obtained on Fig. $5 \mathrm{a}-\mathrm{c}$. At $140 \mathrm{~K}$, the laser emission peak has shifted by $30 \mathrm{meV}$ to lower energy as compared to the laser emission peak at $75 \mathrm{~K}$. Note that the bandgap energy, and thus the optical gain is expected to redshift by typically $10 \mathrm{meV}$ when increasing temperature from 75 to $140 \mathrm{~K}$ (see Fig. S7c of the SI). Lasing at $140 \mathrm{~K}$ is thus observed with the next available confined optical mode that has a better overlap with gain at $140 \mathrm{~K}$, i.e., the $\mathrm{TE}_{9,1}$ mode instead of $\mathrm{TE}_{10,1}$ (Fig. 5c). The $\mathrm{TE}_{9,1}$ mode energy is 

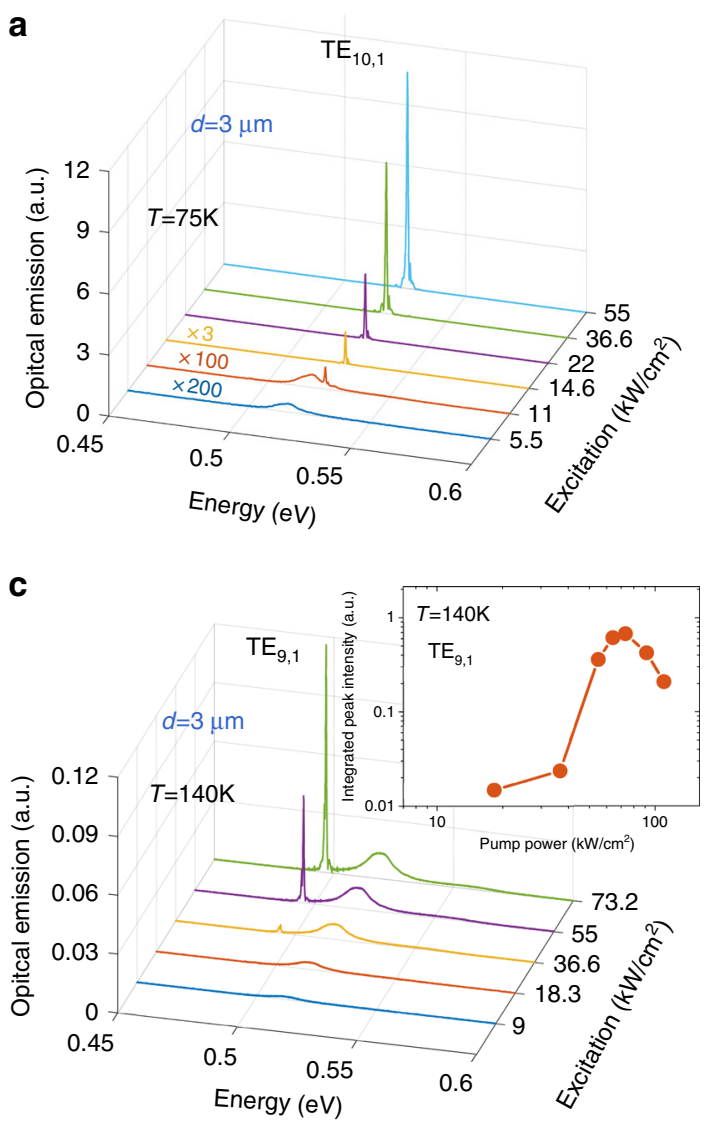

b

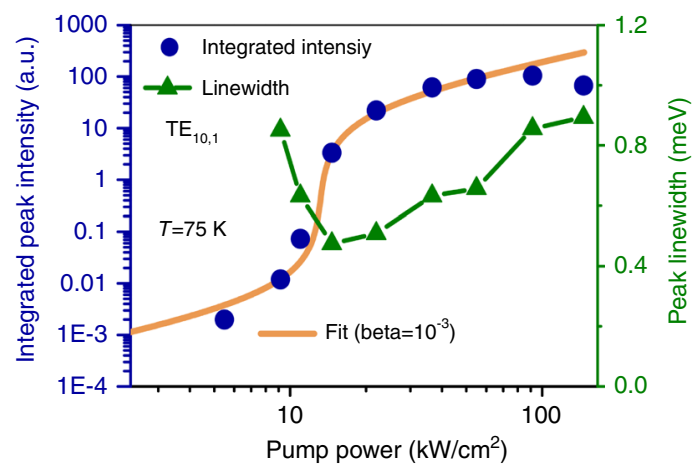

d

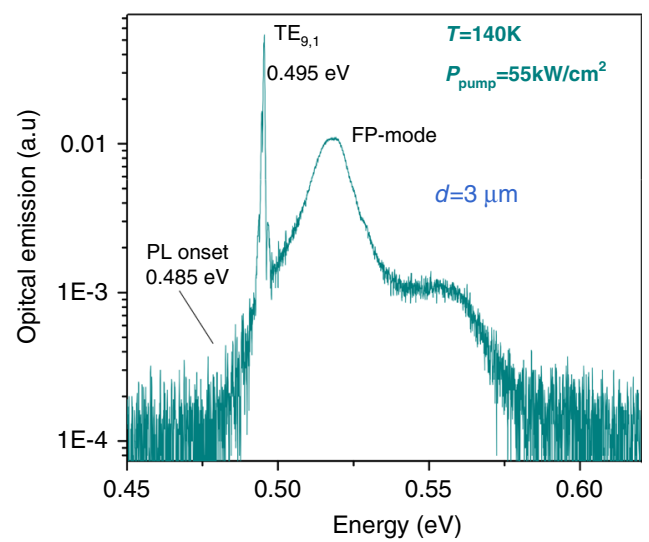

Fig. 5 Down-scaled GeSnOI mesa laser. a Pulsed PL spectra of $3 \mu \mathrm{m}$ diameter GeSnOI mesa with 360-nm-thick GeSn under various excitation densities at $75 \mathrm{~K}$. $\mathbf{b}$ the $L-L$ curve of the laser mode $T E_{10,1}$ at $75 \mathrm{~K}$ in $\log -\log$ scale and mode linewidth as a function of the pump power. $\mathbf{c}$ same as $\mathbf{a}$ at $140 \mathrm{~K}$, the inset shows the $\mathrm{L}-\mathrm{L}$ curve in a log-log scale for the lasing mode which is the $T E_{9,1}$ in this case. $\mathbf{d}$ Emission spectrum above threshold at $140 \mathrm{~K}$ highlighting the $\mathrm{TE}_{9,1}$ lasing mode position with respect to the spontaneous emission. The spontaneous emission is itself strongly modulated by broad peak that stems from Fabry-Pérot (FP) resonance. These FP-modes emit more favorably out-of-plane and are thus better collected than the WGMs

above the onset of spontaneous emission (Fig. 5d). This mode switching when changing the temperature is nonetheless not systematically observed. As discussed in the SI (Fig. S7a, b), we did not observe such mode switching for $400-\mathrm{nm}$-thick disk mesas with $3 \mu \mathrm{m}$ diameter. We can note that the $\mathrm{TE}_{10,1}$ mode for sample with 400-nm-thick GeSn is red-shifted by $7 \mathrm{meV}$ with respect to the $\mathrm{TE}_{10,1}$ mode in the 360 -nm-thick GeSn sample (see Fig. S8 of the SI). With this sample the $\mathrm{TE}_{10,1}$ mode should thus maintain a better spectral overlap with the optical gain than the $\mathrm{TE}_{9,1}$ mode when the temperature increases from 75 to $140 \mathrm{~K}$. The slope drop at high pump intensity as observed in the inset of Fig. $5 \mathrm{c}$ corresponds to the roll-off of the lasers ${ }^{38}$, as a consequence of self-heating and modified carrier distribution spatially and in k-space.

As proof of the feasibility of tuning, in GeSnOI mesas, the laser wavelength and bandgap with external tensile strain, we also probed with pulsed excitation all-around GeSnOI mesas. After the conformal deposition of the top
$\mathrm{SiN}$ stressor layer, an additional etching of the $\mathrm{SiN}$ around the MD was performed to avoid compressive strain injection at the edges of the mesas. In that case, as shown in Fig. 6a, we see a clear redshift of the emission, from 2.4 to $2.8 \mu \mathrm{m}$, be it below and above the lasing threshold. Such a redshift is due to the applied in-plane tensile strain of $0.5 \%$ as deduced from Raman, PL, and FEM analysis.

A more detailed analysis of the lasing performances presented in the SI (section E) however showed that allaround GeSnOI mesas did not have improved lasing performances despite a stronger lifting of the valence band degeneracy, which should have resulted in a transparency threshold reduction. The strain-induced increase of the $\Gamma-\mathrm{L}$ conduction band energy barrier was also expected to improve the maximum lasing temperature. Here, the lasing threshold of $3 \mu \mathrm{m}$ diameter, tensilestrained GeSnOI all-around mesas was $15.5 \mathrm{~kW} / \mathrm{cm}^{2}$, to be compared with $12 \mathrm{~kW} / \mathrm{cm}^{2}$ for quasi-relaxed GeSnOI mesas without $\mathrm{SiN}$ stressors on top. The maximum lasing 

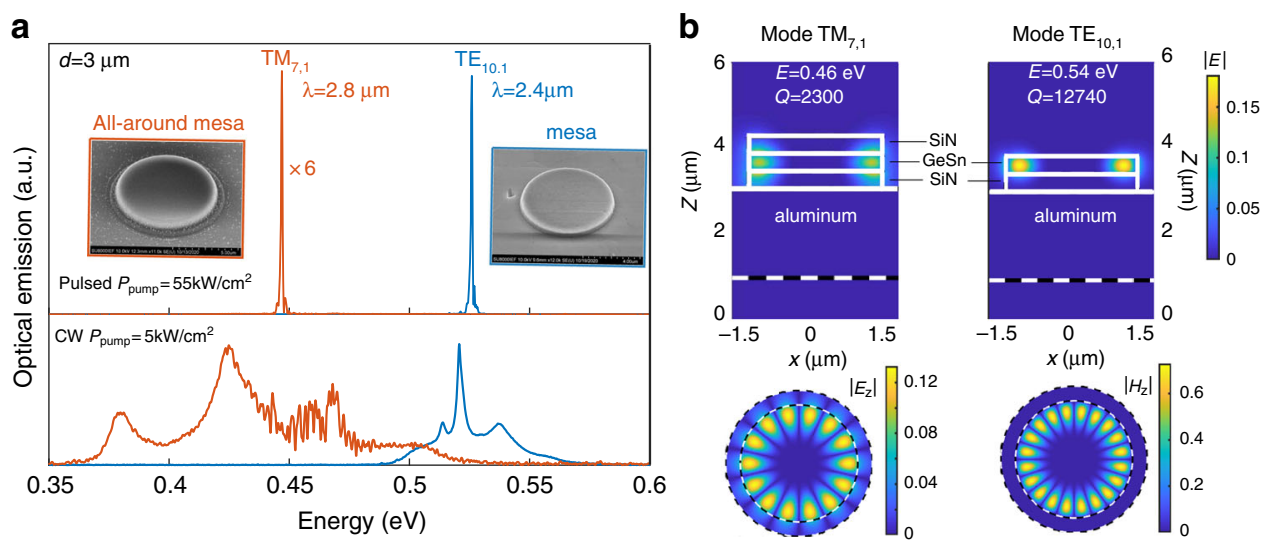

Fig. 6 Tensile strain tuning of GeSnOI laser emission. a Spectra below and above threshold density for $3 \mu$ m diameter GeSnOl mesas with (red curves) or without (blue curves) top SiN stressors. In both cases, the active GeSn layer thickness was $400 \mathrm{~nm}$. b Simulated optical fields and theoretical Q-factor values of $\mathrm{TM}_{7,1}$ and $\mathrm{TE}_{10,1}$ lasing modes for a tensile-strained GeSnOl mesa with a SiN stressor on top and a quasi-relaxed GeSnOI mesa without the top SiN stressor, respectively. Calculations have been performed with an aperiodic Fourier modal method dedicated to body-ofrevolution objects ${ }^{45}$

temperature was also slightly lower, $130 \mathrm{~K}$ for all-around mesas instead of $140 \mathrm{~K}$ without SiN stressors on top (SI section E). The optical gain was thus weaker. Tensilestrained mesas featured TM-polarized optical gain. The lasing mode at $2.8 \mu \mathrm{m}$ of all-around GeSnOI mesas can be assigned the $\mathrm{TM}_{7,1}$ resonance. Since TM-polarized modes, here at a longer wavelength, have a stronger overlap with the $\mathrm{Al}$ metallic layer, it seems likely that the lower performances are due to higher TM losses (Fig. 6b). The Q-factor was of 2300 for the $\mathrm{TM}_{7,1}$ mode, a value significantly lower than the $Q$-factor of 12,740 we had for the $\mathrm{TE}_{10,1}$ laser mode of GeSnOI mesas without $\mathrm{SiN}$ on top. Increasing the bottom SiN thickness, in order to limit the TM field overlap with aluminum and improve the WGM Q-factors, should enable enhanced performances of GeSnOI all-around mesas as shown in the SI (Fig. S4). Aluminum could also be replaced by a dielectric cladding layer to avoid such optical losses. Several strategies using $\mathrm{SiO}_{2}$ layer as dielectric are proposed in the SI (Fig. S5). However, since $\mathrm{SiO}_{2}$ is a poor thermal conductor, one should use thin layers in the order of 100-nm-thick to maintain a good thermal dissipation. Despite degradation of thermal dissipation with thick $\mathrm{SiO}_{2}$ dielectric layers (e.g., $400 \mathrm{~nm}$ ) as replacement of $\mathrm{Al}$, the planar $\mathrm{GeSnOI}$ structure maintains a clear superiority as compared to the suspended GeSn-MD approach for down-scaled geometries (Fig. S5c). Aluminum was selected here as it could be etched selectively with respect to GeSn and $\mathrm{SiN}$ in order to fabricate suspended structures, as in ref. ${ }^{22}$. We show here the interest of using a GeSnOI approach with a bottom stressor that is compatible with an all-around strain transfer scheme without suspending the active GeSn layer, thereby improving thermal dissipation while benefiting from high optical confinement. In the SI
(Fig. S10), we show that, in contrast, the use of a single side $\mathrm{SiN}$ stressor on the top surface of a suspended $\mathrm{MD}^{24,25,34}$ is not suitable for homogeneous strain injection into the GeSn active layer. Such a single stressor approach indeed resulted in optical gain quenching.

MD mesas with SiN stressors have many advantages as optical laser cavities, such as scalability with the operating wavelength and high $Q$-factor provided by WGMs. One of their drawbacks, though, is the in-plane circular spread of the WGM radiation pattern. It differs markedly from the auto-collimated beam from conventional lasers, making emitted power collection tricky. The two common approaches to extract power are (i) side or top collection of radiated power with optical apparatus such as microscope objectives (ii) evanescent coupling of the disk WGM with expanded waveguide modes. The first does not yield a total collection of the in-plane radiation pattern, due to the limited numerical aperture of the collecting objectives. The second approach, with addition of some process complexity, requires an optimization of light injection into the waveguide. Critical coupling conditions have to be met by fine-tuning the gap between waveguides and MDs. Such an evanescent coupling also occurs over a limited fraction of the disk periphery and has an impact on the cavity $Q$-factor and therefore on its lasing properties. We show here that GeSnOI mesas are advantageous for a proper light collection. Indeed, circular diffraction gratings can be added around disk-shape mesas to redirect the light vertically.

Here, we collect the MD emission vertically with an $\times 50$ objective with a 0.65 numerical aperture. Light is thus collected from the sample surface within a solid angle of $40^{\circ}$. Yet, standard MDs have a radiation pattern which is mainly out of this solid angle, as shown in Fig. 7a, with a 

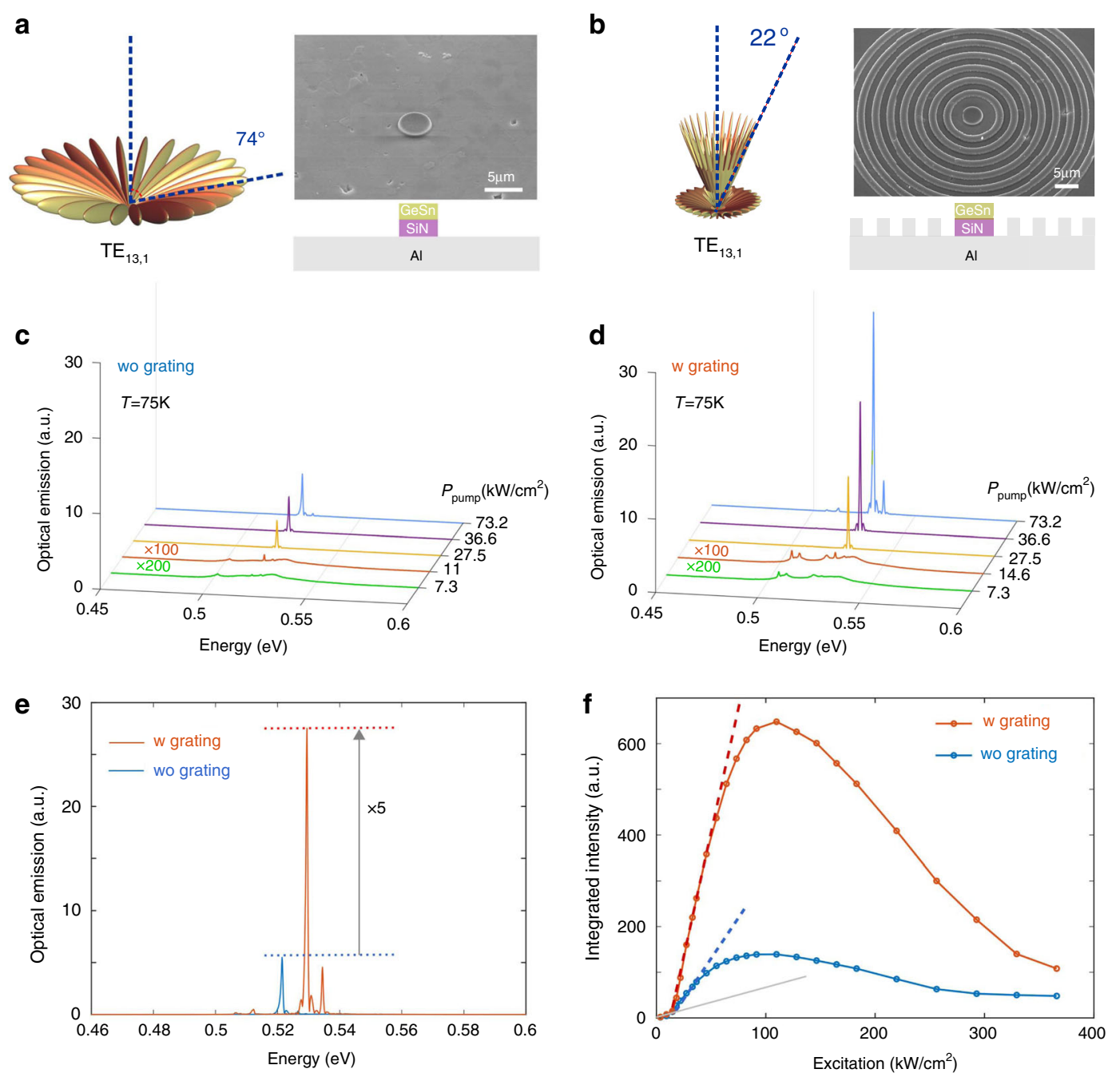

Fig. 7 Vertical out-coupling of GeSnOI mesa lasing WGM. a, b SEM images and calculated emission patterns of $4 \mu \mathrm{m}$ diameter GeSnOI mesas without and with Al circular grating (7 rings spaced by $3 \mu \mathrm{m}$ with a duty cycle of 50\%). Calculations have been performed with an aperiodic Fourier modal method dedicated to body-of-revolution objects ${ }^{45} \cdot \mathbf{c}$, d Power-dependent spectra without and with Al circular grating. e Spectra with and without grating for a $73.2 \mathrm{~kW} / \mathrm{cm}^{2}$ excitation density. $\mathbf{f} \mathrm{L}-\mathrm{L}$ curves of $\mathrm{GeSnOl}$ lasers with and without grating

modeling of the WGM radiation for a $4 \mu \mathrm{m}$ diameter GeSnOI mesa MD. A small MD diameter was chosen as it simplified the modal analysis. Such a mesa features a monomode lasing at $0.52 \mathrm{eV}$ which can be assigned to the $\mathrm{TE}_{13,1}$ mode at an energy of $0.54 \mathrm{eV}$. The maximum radiation angle is situated around $74^{\circ}$ with respect to the normal incidence (Fig. 7a). These simulations also show an extremely small coupling factor from such WGM pattern to the collecting objective, of the order of $10^{-5}$ only. The collected emission from WGM lasing therefore comes from out-of-plane leakage rather than from radiative emission. Such leakage paths stem from imperfection introduced during fabrication (sidewall and surface roughness, circular symmetry breaking). These imperfections, useful to collect more light into the collection angle, are obviously unwanted in practice since they result in lower $Q$-factors and increase lasing thresholds.

One way of overcoming this issue would be to have an optimized process flow in order to reach the highest $Q$ factors possible and use external gratings to redirect the WGM in-plane radiation pattern. This is feasible with GeSnOI mesas, while it would be very tricky with GeSn suspended MDs. We thus designed a circular diffraction grating with optimal period and duty cycle to maximize the redirection of the WGMs light into the $40^{\circ}$ solid angle for a $4 \mu \mathrm{m}$ diameter GeSnOI mesa (see the SI). Figure $7 \mathrm{~b}$ radiation diagram with such additional circular grating is drastically modified for the $\mathrm{TE}_{13,1}$ mode, with then a maximal radiation angle of $22^{\circ}$ compared to normal incidence. The total flux integrated into our objective collection angle becomes theoretically of $30 \%$ as 
compared to $2.4 \times 10^{-5}$ without grating. Such a power collection improvement is expected for lasing WGM mode specifically coupled to the grating. The GeSnOI mesa laser emission spectra and integrated collected intensity, below and above laser threshold, are shown in Fig. 7c-f with and without grating. Below the threshold, spontaneous emission dominates the spectrum and the contribution from WGM is weak as it is not amplified. Intensities with and without grating are thus equivalent. On the contrary, above a threshold which was around $15 \mathrm{~kW} / \mathrm{cm}^{2}$ in both cases, the WGM contribution dominates the spectrum and a clear improvement of collected intensity is observed, typically by a factor of 5 for mesas with gratings. The maximum collected peak power is around $20-30 \mu \mathrm{W}$.

The modeling predicted an improvement by 12,500 of the intensity collected by the objective with such a grating. It is thus very clear that initially, the collected light without the grating mainly stems from leak paths, as mentioned above, due to roughness scattering not accounted for in the modeling. The very weak estimated coupling factor of the WGM radiation to the objective, of the order of $10^{-5}$ for mesa MDs, is thus not representative of the power collected experimentally. Such scattering obviously tends to reduce the cavity quality factor of the $\mathrm{TE}_{13,1}$ mode calculated to be $Q_{\mathrm{rad}}=8160$ for perfectly smooth surfaces and sidewalls. Such a method would thus yield a better control of the total power radiated from the cavity without changing the cavity geometry and its lasing characteristics. The same emission control is feasible for larger diameter cavities. The $4 \mu \mathrm{m}$ diameter featuring single-mode lasing was chosen here to simplify the experimental analysis, but similar results with higher diameter disks have been simulated (data not shown). Gratings without aluminum could as well be envisaged.

\section{Discussion}

On the basis of our proposed GeSnOI platform, one could also obtain in-plane coupling of radiated emission into waveguides if the aim was to inject the laser emission into a photonic circuit. The most obvious way would be to use $\mathrm{SiN}$, which offers a high optical index and high transparency in the mid-infrared, as the waveguide material. SiN was indeed proven to be suitable for mid-IR photonic circuits in the literature $\mathrm{e}^{39,40}$ and could be combined with $\mathrm{GeSn}$ as active materials. This should be feasible with our proposed GeSnOI stack. Selective deposition of SiN could be used if an increase in the waveguide core thickness in some places was needed. Blanket SiN deposition followed by selective etching of some of it in specific places, as used here to fabricate allaround mesas, could be selected to construct the in-plane photonic circuit. SiN etching can for instance be performed using fluoride-based plasma, which is very selective to $\mathrm{GeSn}^{41}$. The aluminum layer might have to be replaced by low index dielectrics cladding such as silicon dioxide, then ${ }^{42}$. As discussed above and detailed in the SI (section $\mathrm{C}$ ), the replacement of the $\mathrm{Al}$ layer requires specific designs with the aim to maintain a good thermal dissipation for the laser devices. We note that the mesa structure offers superior thermal dissipation than underetched GeSn/Ge SRB layer while having equivalent optical confinement factors. In this work, we used $\mathrm{Al}$ grating for light beam engineering with GeSnOI. There are many others possibilities, using dielectric instead of $\mathrm{Al}$ material for that purpose when using infrared compatible materials. In the latter cases, the periodicity and the dimension will depend on the optical parameters of the chosen material. We can further envision more complex mode radiation shaping as compared to the one provided in the present work, by using phase engineering of the wave fronts.

We have shown here the interest of using GeSn active layers bonded on dielectric stressors to fabricate GeSnOI stacks, resulting in optimized group-IV laser designs in terms of defects, strain, modal, and thermal engineering. Additionally, bandgap energy and directness parameters could be tuned via tensile strain. The strain can be varied by using different parameters for the $\mathrm{SiN}$ stressor deposition, by varying the $\mathrm{Sn}$ content of the alloy, and the degree of relaxation of the GeSn layer through the conditions of epitaxial growth. Obviously, such an approach can be applied to different GeSn active layers with varying Sn contents, as well as complex heterostructures with quantum wells, to provide additional electronic band and gain engineering. The first purpose of the present work was to show the relevance of GeSnOI MD mesas compared to more conventional structures such as suspended GeSn MDs on Ge pillars. The same as-grown 500-nmthick GeSn $10.5 \%$ layer was used for such a comparison. GeSnOI mesas have approximately the same high index contrast than suspended MDs and are suitable for the fabrication of planar structures, free from interface defects, such as ridge Fabry-Perot waveguides, ring cavities, or even photonic crystals. Enhanced performances were obtained with the GeSnOI approach as compared to conventional approaches. We highlighted that planar emission can be redirected vertically thanks to additional gratings, illustrating the flexibility provided by such a technology.

The key advantage of this GeSnOI platform is its ability to combine active laser structures with passive $\mathrm{SiN}$ circuitry from the near-infrared to the mid-infrared. It represents a new paradigm for infrared Group-IV photonics that eliminates the need for III-V laser integration.

To be able to reach temperature with this GeSnOI approach, one of the most obvious way is to increase the tin content of the alloy. Here we have reported a complete 
study as a first proof of concept to assess the GeSnOI laser technology with respect to the conventional $\mathrm{GeSn} / \mathrm{Ge}$ $\mathrm{SRB}$, starting from a GeSn with a $10.5 \%$ tin content. It has been shown in the literature that lasing up to $270 \mathrm{~K}$ can be reached in suspended structures, thus with poor thermal management, using under-etched GeSn/Ge SRB cavities $^{32}$. In this case, a tin content of $16 \%$ in the active region was used. The increase of tin content induces an increase of the directness of the band structure that is one of the main key ingredient which allowed for the highertemperature laser operation as compared with lower tin content materials. As the GeSnOI approach exhibits improved performances, we can thus expect that this approach, if applied to materials with higher tin content than in this work, could lead to even higher temperatures of laser operation.

One second challenging goal is to demonstrate electrically driven devices. We emphasize that this is completely compatible with our approach if we start with GeSn pin junctions since the bonding can be applied to any kind of $\mathrm{GeSn}$ heterostructures. Bonding GeSn diodes is completely feasible and the electrical injection can be made with such GeSnOI active layers. The GeSnOI can even offer better performances as compared to a standard approach since the high index contrast offers more flexibilities in the design to reduce the overlap of the laser modes with doped contacts and the electrodes. It has been shown that electrical injection in optical microcavities is compatible with the use of the SiN stressor transfer method, as long as specific designs are used to define the electrode geometries ${ }^{43}$.

\section{Materials and methods}

\section{GeSn on Ge SRB material growth on silicon}

Epitaxial growth of close to 500 -nm-thick GeSn $10.5 \%$ was performed in a $200 \mathrm{~mm}$ Epi Centura 5200 cluster tool from Applied Materials on a Ge SRB on silicon. The Ge SRB was grown with $\mathrm{GeH}_{4}$ and a low temperature/high temperature approach, followed by some short duration Thermal cycling to reduce the threading dislocations densities to values around $10^{7} \mathrm{~cm}^{-2}$, typically. Meanwhile, the GeSn layer was grown close to $337^{\circ} \mathrm{C}$ at 100 Torr thanks to $\mathrm{Ge}_{2} \mathrm{H}_{6}$ and $\mathrm{SnCl}_{4}$ precursors ${ }^{44}$. The as-grown layers was analyzed by $\mathrm{X}$-ray diffraction reciprocal space mapping. The layer has a residual compressive strain of $-0.5 \%$.

\section{WGM radiation modeling}

The WGM analysis and their scattering by the $\mathrm{Al}$ grating was performed in the framework of the aperiodic Fourier modal method that was detailed in ref. ${ }^{45}$. The permittivity model considered, here and in previous sections, for aluminum was a realistic Lorentz-Drude model ${ }^{46}$. More details are provided in the SI (section G) for the design of the $\mathrm{Al}$ grating.

\section{Acknowledgements}

This work was supported by the French RENATECH network, the French National Research Agency (Agence Nationale de la Recherche, ANR) through funding of the ELEGANTE project (ANR-17-CE24-0015). B.W. was supported by Nano2022 IPCEI project with STMicroelectronics.

\section{Author details}

'Université Paris-Saclay, CNRS, C2N, 10 boulevard Thomas Gobert, 91120 Palaiseau, France. ${ }^{2}$ Université Côte d'Azur, CNRS, CRHEA, Rue Bernard Grégory, 06905 Sophia-Antipolis, France. ${ }^{3}$ STMicroelectronics, Rue Jean Monnet, 38054 Crolles, France. ${ }^{4}$ Université Grenoble Alpes, CEA, IRIG-DePhy, 17 rue des Martyrs, 38000 Grenoble, France. ${ }^{5}$ Université Grenoble Alpes, CEA, Leti, 17 rue des Martyrs, 38000 Grenoble, France

\section{Conflict of interest}

The authors declare no competing interests.

Supplementary information The online version contains supplementary material available at https://doi.org/10.1038/s41377-021-00675-7.

Received: 11 May 2021 Revised: 25 October 2021 Accepted: 29 October 2021

Published online: 17 November 2021

\section{References}

1. Soref, R., Buca, D. \& Yu, S. Q. Group IV photonics: driving integrated optoelectronics. Opt. Photonics News 27, 32-39 (2016).

2. Thomson, D. et al. Roadmap on silicon photonics. J. Opt. 18, 073003 (2016).

3. Wang, Z. C. et al. Novel light source integration approaches for silicon photonics. Laser Photonics Rev. 11, 1700063 (2017).

4. Seifried, M. et al. Monolithically integrated CMOS-compatible III-V on silicon lasers. IEEE J. Sel. Top. Quantum Electron. 24, 8200709 (2018).

5. Elbaz, A. et al. Reduced lasing thresholds in GeSn microdisk cavities with defect management of the optically active region. ACS Photonics 7, 2713-2722 (2020).

6. Zhou, Y. Y. et al. Electrically injected GeSn lasers on Si operating up to $100 \mathrm{~K}$. Optica 7, 924-928 (2020).

7. Zhou, Y. Y. et al. Optically pumped GeSn lasers operating at $270 \mathrm{~K}$ with broad waveguide structures on Si. ACS Photonics 6, 1434-1441 (2019).

8. Wirths, S. et al. Lasing in direct-bandgap GeSn alloy grown on Si. Nat. Photonics 9, 88-92 (2015).

9. Singh, V. et al. Mid-infrared materials and devices on a Si platform for optical sensing. Sci. Technol. Adv. Mater. 15, 014603 (2014).

10. Hodgkinson, J. \& Tatam, R. P. Optical gas sensing: a review. Meas. Sci. Technol. 24, 012004 (2013).

11. Reboud, V. et al. Optically pumped GeSn micro-disks with $16 \%$ Sn lasing at $3.1 \mu \mathrm{m}$ up to 180 K. Appl. Phys. Lett. 111, 092101 (2017).

12. Al-Kabi, S. et al. An optically pumped $2.5 \mu \mathrm{m}$ GeSn laser on Si operating at 110 K. Appl. Phys. Lett. 109, 171105 (2016).

13. Dou, W. et al. Investigation of GeSn strain relaxation and spontaneous composition gradient for low-defect and high-Sn alloy growth. Sci. Rep. 8, 5640 (2018).

14. von den Driesch, N. et al. Direct bandgap group IV epitaxy on Si for laser applications. Chem. Mater. 27, 4693-4702 (2015).

15. Gupta, S. et al. Achieving direct band gap in germanium through integration of Sn alloying and external strain. J. Appl. Phys. 113, 073707 (2013).

16. Pezzoli, F. et al. Temperature-dependent photoluminescence characteristics of GeSn epitaxial layers. ACS Photonics 3, 2004-2009 (2016).

17. Stange, D. et al. Short-wave infrared LEDs from GeSn/SiGeSn multiple quantum wells. Optica 4, 185-188 (2017).

18. Stange, D. et al. GeSn/SiGeSn heterostructure and multi quantum well lasers. ACS Photonics 5, 4628-4636 (2018).

19. Grant, P. C. et al. Direct bandgap type-l GeSn/GeSn quantum well on a GeSnand Ge- buffered Si substrate. AIP Adv. 8, 025104 (2018).

20. Thai, Q. M. et al. GeSn heterostructure micro-disk laser operating at 230 K. Opt. Express 26, 32500-32508 (2018).

21. Du, W. et al. Study of Si-based GeSn optically pumped lasers with micro-disk and ridge waveguide structures. Front. Phys. 7, 147 (2019). 
22. Elbaz, A. et al. Ultra-low-threshold continuous-wave and pulsed lasing in tensile-strained GeSn alloys. Nat. Photonics 14, 375-382, https://doi.org/ 10.1038/s41566-020-0601-5 (2020).

23. Thai, Q. M. et al. GeSn optical gain and lasing characteristics modelling. Phys. Rev. B 102, 155203 (2020).

24. Rainko, D. et al. Impact of tensile strain on low Sn content GeSn lasing. Sci. Rep. 9, 259 (2019).

25. Ghrib, A. et al. Tensile-strained germanium microdisks. Appl. Phys. Lett. 102 221112 (2013)

26. Ghrib, A. et al. All-around SiN stressor for high and homogeneous tensile strain in germanium microdisk cavities. Adv. Opti. Mater. 3, 353-358 (2015).

27. Elbaz, A. et al. Germanium microlasers on metallic pedestals. APL Photonics $\mathbf{3}$, 106102 (2018)

28. Armand Pilon, F. T. et al. Lasing in strained germanium microbridges. Nat. Commun. 10, 2724 (2019).

29. Süess, M. J. et al. Analysis of enhanced light emission from highly strained germanium microbridges. Nat. Photonics 7, 466-472 (2013).

30. Nam, D. et al. Study of carrier statistics in uniaxially strained Ge for a lowthreshold Ge laser. IEEE J. Sel. Top. Quantum Electron. 20, 16-22 (2014).

31. Imbrenda, D. et al. Infrared dielectric response, index of refraction, and absorption of germanium-tin alloys with tin contents up to $27 \%$ deposited by molecular beam epitaxy. Appl. Phys. Lett. 113, 122104 (2018).

32. Chrétien, J. et al. GeSn lasers covering a wide wavelength range thanks to uniaxial tensile strain. ACS Photonics 6, 2462-2469 (2019).

33. Elbaz, A. et al. Solving thermal issues in tensile-strained Ge microdisks. Opt. Express 26, 28376-28384 (2018)

34. Moutanabbir, O. et al. Monolithic infrared silicon photonics: the rise of (Si)GeSn semiconductors. Appl. Phys. Lett. 118, 110502 (2021).
35. Tabataba-Vakili, F. et al. Analysis of low-threshold optically pumped III-nitride microdisk lasers. Appl. Phys. Lett. 117, 121103 (2020).

36. Baba, T. \& Sano, D. Low-threshold lasing and Purcell effect in microdisk lasers at room temperature. IEEE J. Sel. Top. Quantum Electron. 9, 1340-1346 (2003).

37. Baba, T. et al. Spontaneous emission factor of a microcavity DBR surfaceemitting laser. IEEE J. Quantum Electron. 27, 1347-1358 (1991).

38. Piprek, J., White, J. K. \& SpringThorpe, A. J. What limits the maximum output power of long-wavelength AlGalnAs/InP laser diodes? IEEE J. Quantum Electron. 38, 1253-1259 (2002)

39. Mashanovich, G. Z. et al. Low loss silicon waveguides for the mid-infrared. Opt. Express 19, 7112-7119 (2011)

40. Miller, S. A. et al. Low-loss silicon platform for broadband mid-infrared photonics. Optica 4, 707-712 (2017).

41. Gupta, S. et al. Highly selective dry etching of germanium over germanium-tin (Ge1-xSnx): a novel route for Ge1-xSnx nanostructure fabrication. Nano Lett. 13, 3783-3790 (2013).

42. Lin, P. T. et al. Planar silicon nitride mid-infrared devices. Appl. Phys. Lett. 102, 251121 (2013).

43. Prost, $M$. et al. Tensile-strained germanium microdisk electroluminescence. Opt. Express. 23, 6722-6730 (2015).

44. Aubin, J. et al. Growth and structural properties of step-graded, high Sn content GeSn layers on Ge. Semicond. Sci. Technol. 32, 094006 (2017).

45. Bigourdan, F., Hugonin, J.-P. \& Lalanne, P. Aperiodic-Fourier modal method for analysis of body-of-revolution photonic structures. J. Opt. Soc. Am. A 31, 1303-1311 (2014)

46. Rakić, A. D. et al. Optical properties of metallic films for vertical-cavity optoelectronic devices. Appl. Opt. 37, 5271-5283 (1998). 